\title{
Soap opera in the maze: Geometry matters in Marangoni flows
}

\author{
Fernando Temprano-Coleto, ${ }^{1, *}$ François J. Peaudecerf, ${ }^{2}$ Julien R. Landel, ${ }^{3}$ Frédéric Gibou,,${ }^{1,4}$ \\ and Paolo Luzzatto-Fegiz ${ }^{1}$ \\ ${ }^{1}$ Department of Mechanical Engineering, University of California Santa Barbara, Santa Barbara, \\ California 93106, United States \\ ${ }^{2}$ Department of Civil, Environmental, and Geomatic Engineering, ETH Zürich, 8093 Zürich, Switzerland \\ ${ }^{3}$ School of Mathematics, Alan Turing Building, University of Manchester, Oxford Road, \\ Manchester M13 9PL, United Kingdom \\ ${ }^{4}$ Department of Computer Science, University of California Santa Barbara, Santa Barbara, \\ California 93106, United States
}

(Received 2 February 2018; published 17 October 2018)

\begin{abstract}
This paper is associated with a video winner of a 2017 APS/DFD Gallery of Fluid Motion Award. The original video is available from the Gallery of Fluid Motion, https://doi.org/10.1103/APS.DFD.2017.GFM.V0098
\end{abstract}

DOI: 10.1103/PhysRevFluids.3.100507

The ancient Greek myth of Theseus and the Minotaur is perhaps the most famous example of the fascination that maze solving has historically triggered in humankind. Maze-solving strategies also have important practical applications, including path finding in robotics [1], urban transportation [2], and cognitive neuroscience [3]. Since the digital computation of the shortest path across a maze is efficient for simple cases only [4,5], numerous more unconventional, analog methods have been suggested in the past few decades. For instance, amoeboid organisms are able to solve mazes when a suitable gradient of nutrients is present [6], and chemical waves have been reported to find optimal pathways in complex labyrinths [7]. Other examples among these analog maze-solving techniques rely on fluid-mechanical effects, such as exploiting a pressure gradient between the inlet and outlet of a complex microfluidic network in order to drive a dyed fluid between the two [8].

We demonstrate here that the Marangoni effect produced by a surfactant is a fast and highly efficient mechanism of maze solving. We present the experimental observation of a small amount of dilute soap, which successfully solves a maze filled with milk with minimal penetration into side branches. While maze solving using thermal [9], pH-induced [10,11], and surfactant-generated [12] Marangoni flows have previously been reported, in those cases the motion is established in the maze by introducing an alteration at the outlet, with the tracer particles being drawn from the inlet by the resulting flow, such that a tracer anywhere in the maze is also transported towards the exit. However, in the present work the decrease in surface tension originates at the inlet, and the tracer follows the correct path to the outlet without any pre-existing gradient of surface tension ahead of its path.

The experiment consists of a square plate of acrylic $(127 \times 127 \mathrm{~mm})$ with a series of open channels approximately $3 \mathrm{~mm}$ deep and forming a small maze. First, the maze is filled with milk [13], and a drop of red dye is added at the inlet reservoir, acting as a passive tracer. Subsequently, a brush previously

\footnotetext{
*Corresponding author: ftempranocoleto@ucsb.edu
}

Published by the American Physical Society under the terms of the Creative Commons Attribution 4.0 International license. Further distribution of this work must maintain attribution to the author(s) and the published article's title, journal citation, and DOI. 

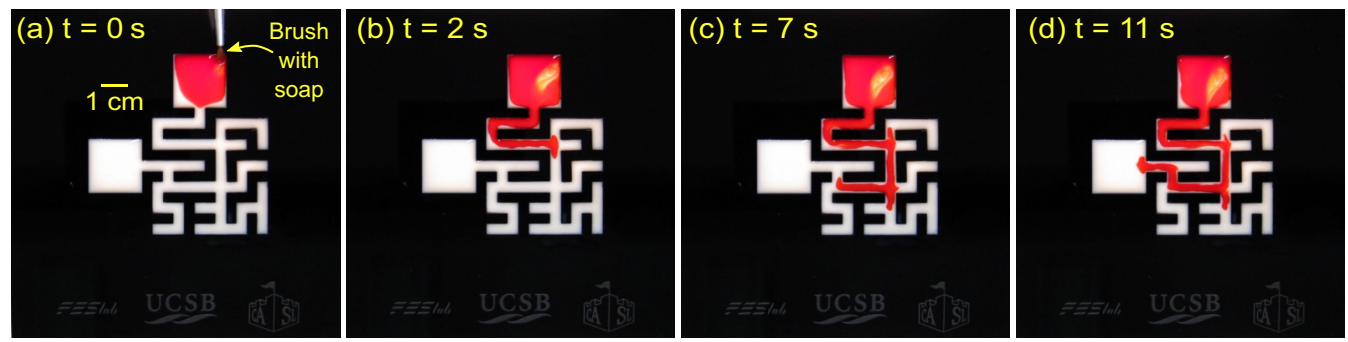

FIG. 1. Snapshots of a $0.2 \%$ solution of soap solving a small maze filled with milk, from time $t=0 \mathrm{~s}$ until $t=11 \mathrm{~s}$, when the red dye reaches the exit. The width of the channels is $4 \mathrm{~mm}$. DOI: https://doi.org/10.1103/APS.DFD.2017.GFM.V0098

immersed in a soap solution ( $0.2 \%$ volume, Seventh Generation brand) is briefly introduced in the milk at the inlet, for a time not exceeding $1 \mathrm{~s}$ [Figs. 1(a) and 2(a)]. Immediately after the brush is introduced, a Marangoni flow away from it is triggered, due to the locally lower surface tension of the milk-air interface in the area with soap, which overlaps with the dye.

The dyed milk, entrained by the soap, barely penetrates into side channels with dead ends, opting for the right path toward the outlet at every bifurcation until it solves the maze [Figs. 1(b)-1(d) and 2(b)-2(f)]. This fact may appear surprising since, as mentioned above, the front of red dye does not advance following a gradient of surface tension originated from the outlet. However, it could potentially be explained by the presence of endogenous surfactants, trace amounts of other surfaceactive substances that are naturally unavoidable in the fluid. These small quantities of surfactant are usually insufficient to produce a noticeable decrease of surface tension in the initial fluid, but can
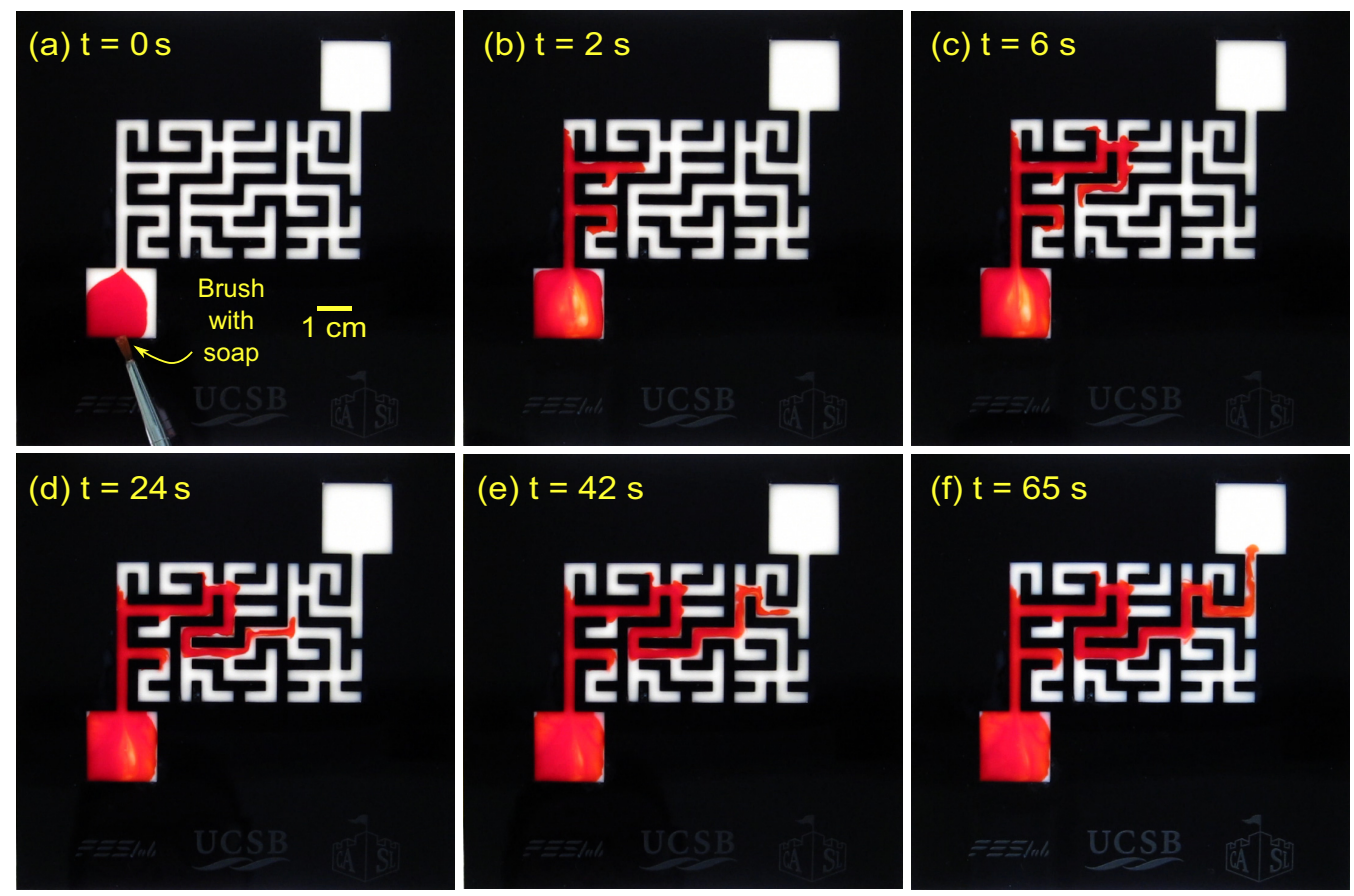

FIG. 2. Snapshots of a $0.2 \%$ solution of soap solving a larger and more complex maze, also filled with milk. The red dye used as tracer reaches the exit at time $t=60 \mathrm{~s}$. The width of the channels is $3 \mathrm{~mm}$. DOI: https://doi.org/10.1103/APS.DFD.2017.GFM.V0098 
have a marked effect on the dynamics, described as follows. As the front of dye spreads across the maze, it compresses the interface ahead, thus increasing the concentration of endogenous surfactant and creating a Marangoni force opposing the motion, eventually stopping the flow. This effect has been reported to occur in the spreading of surfactants in a thin film of liquid [14], and could explain the maze-solving effect here. Indeed, when reaching a bifurcation the dye would naturally choose the path of least resistance, which is the one with the largest surface area ahead, since it would minimize the downstream concentration of endogenous surfactants. According to this hypothesis, the relatively large surface area of the outlet reservoir of the maze offers the lowest resistance at every bifurcation, thus enabling the soap to solve the maze correctly.

This result is a striking example of the dramatic consequences of surface-active impurities in fluid flows. Their role in slowing down the rising motion of bubbles in a liquid [15] has been known for decades [16]. Very recently, it has been shown experimentally that trace amounts of surfactants can also critically hinder the drag reduction ability of superhydrophobic surfaces [17]. Taking these effects into account can therefore be essential in the study and prediction of flows involving fluid interfaces.

This work has been supported by the University of California Santa Barbara Academic Senate and California NanoSystems Institute Challenge Grant, as well as by ONR MURI N00014-17-1-2676. We gratefully acknowledge conversations with Oliver Jensen, who pointed us to works on endogenous surfactant.

[1] N. S. V. Rao, S. Kareti, W. Shi, and S. S. Iyengar, Robot navigation in unknown terrains: Introductory survey of non-heuristic algorithms, ORNL/TM-12410, Oak Ridge National Laboratory, Oak Ridge, TN, 1993.

[2] P. Modesti and A. Sciomachen, A utility measure for finding multiobjective shortest paths in urban multimodal transportation networks, Eur. J. Oper. Res. 111, 495 (1998).

[3] D. A. Crowe, B. B. Averbeck, M. V. Chafee, J. H. Anderson, and A. P. Georgopoulos, Mental maze solving, J. Cogn. Neurosci. 12, 813 (2000).

[4] E. W. Dijkstra, A note on two problems in connexion with graphs, Numer. Math. 1, 269 (1959).

[5] J. Tsitsiklis, Efficient algorithms for globally optimal trajectories, IEEE Trans. Autom. Control 40, 1528 (1995).

[6] T. Nakagaki, H. Yamada, and Á. Tóth, Maze-solving by an amoeboid organism, Nature (London) 407, 470 (2000).

[7] O. Steinbock, Á. Tóth, and K. Showalter, Navigating complex labyrinths: Optimal paths from chemical waves, Science 267, 868 (1995).

[8] M. J. Fuerstman, P. Deschatelets, R. Kane, A. Schwartz, P. J. A. Kenis, J. M. Deutch, and G. M. Whitesides, Solving mazes using microfluidic networks, Langmuir 19, 4714 (2003).

[9] P. Lovass, M. Branicki, R. Tóth, A. Braun, K. Suzuno, D. Ueyama, and I. Lagzi, Maze solving using temperature-induced Marangoni flow, RSC Adv. 5, 48563 (2015).

[10] K. Suzuno, D. Ueyama, M. Branicki, R. Tóth, A. Braun, and I. Lagzi, Maze solving using fatty acid chemistry, Langmuir 30, 9251 (2014).

[11] K. Suzuno, D. Ueyama, M. Branicki, R. Tóth, A. Braun, and I. Lagzi, Marangoni flow driven maze solving, in Advances in Unconventional Computing, Vol. 2: Prototypes, Models and Algorithms, edited by A. Adamatzky (Springer International Publishing, Cham, 2017), pp. 237-243.

[12] Y. Wang, X. Liu, X. Li, J. Wu, Y. Long, N. Zhao, and J. Xu, Directional and path-finding motion of polymer hydrogels driven by liquid mixing, Langmuir 28, 11276 (2012).

[13] The choice of milk is for visualization purposes due to the high contrast with the red dye. The same maze-solving effect is observed if water is used as the base fluid. 
[14] J. B. Grotberg, D. Halpern, and O. E. Jensen, Interaction of exogenous and endogenous surfactant: Spreading-rate effects, J. Appl. Physiol. 78, 750 (1995).

[15] R. Palaparthi, D. T. Papageorgiou, and C. Maldarelli, Theory and experiments on the stagnant cap regime in the motion of spherical surfactant-laden bubbles, J. Fluid Mech. 559, 1 (2006).

[16] V. G. Levich and V. S. Krylov, Surface-tension-driven phenomena, Annu. Rev. Fluid Mech. 1, 293 (1969).

[17] F. J. Peaudecerf, J. R. Landel, R. E. Goldstein, and P. Luzzatto-Fegiz, Traces of surfactants can severely limit the drag reduction of superhydrophobic surfaces, Proc. Natl. Acad. Sci. USA 114, 7254 (2017). 